\title{
Desempenho produtivo de Pseudoplatystoma corruscans estocados em sistemas de criação: semi-intensivo (viveiro escavado) e intensivo (tanque-rede)
}

\author{
Growth performance of Pseudoplatystoma corruscans stocked in rearing systems: \\ semi-intensive (ponds) and intensive (cages)
}

\section{Andressa Daniela de Sousa Liranço ${ }^{\mathrm{I}}$ Elizabeth RomagosaII João Donato Scorvo-Filho ${ }^{\mathrm{III}}$}

\section{RESUMO}

Objetivou-se comparar o desempenho produtivo $e$ custos de produção de exemplares de pintado, Pseudoplatystoma corruscans, estocados em dois sistemas de criação: semi-intensivo (viveiro escavado, VE) e intensivo (tanque-rede, TR). Trezentos (300) peixes, com um ano de idade, foram estocados, sendo 150 em um VE (médias de peso e comprimento: $1,48 \pm 0,46 \mathrm{~kg}$ e $57,31 \pm 6,42 \mathrm{~cm}$ ) e 150 divididos em três TR (médias de peso e comprimento: $1,27 \pm 0,34 \mathrm{~kg} e$ $55,05 \pm 4,11 \mathrm{~cm}$ ). Foram alimentados com ração extrusada de $15 \mathrm{~mm}$ (diâmetro) $40 \% \mathrm{~PB}$ e $3110 \mathrm{kcal} \mathrm{ED} \mathrm{kg}^{-1}$, ajustada mensalmente à quantidade de ração. Os parâmetros físicoquímicos da água, observados durante o experimento, foram temperatura $=24,08^{\circ} \mathrm{C} \pm 3,23 ; p H=6,89 \pm 0,39$ e oxigênio dissolvido $=7,57 \pm 0,97 \mathrm{mg} \mathrm{L}^{-1}$. Os reultados obtidos dos valores médios finais dos comprimentos ( $V E=74,07 \pm 4,34 \mathrm{~cm}$; $T R=70,33 \pm 5,02 \mathrm{~cm})$ e pesos dos peixes $(V E=3,41 \pm 0,58 \mathrm{~kg} e$ $T R=2,94 \pm 0,60 \mathrm{~kg}$ ) indicaram desempenho semelhante nos dois sistemas. As médias do fator de condição (0,09-0,036); ganho em peso diário $\left(9,29 \mathrm{~g} \mathrm{dia} \mathrm{di}^{-1}-8,95 \mathrm{~g} \mathrm{dia}^{-1}\right)$; conversão alimentar (3,09-4,15); consumo total de ração $\left(29,60 \mathrm{~g}\right.$ dia $^{-1}-74,16 \mathrm{~g}$ dia $\left.^{-1}\right)$; índice de crescimento $(0,219-0,215)$ e sobrevivência (97,33-90,67\%) para VE e TR, respectivamente. Houve interação significativa entre os sistemas de criação e mês $(P<0,05)$. O quilo de peixe produzido foi de $R \$ 8,76$ (US\$ $2,85)$ e $R \$ 8,73$ (US\$ 2,33) para o VE e TR, respectivamente. Embora o VE tenha demonstrado melhor desenvolvimento durante o período e uma vantagem econômica, o indice de crescimento mostrou que ambos os sistemas tiveram desempenhos semelhantes.

Palavras-chave: desempenhos zootécnicos e econômicos, Pseudoplatystoma corruscans.

\begin{abstract}
The objective of this study was to compare the growth performance and cost of production of Pseudoplatystoma corruscans stocked in two culture systems: semi-intensive (ponds, $P$ ) and intensive (cage, C). From three hundred (300) oneyear-old fish, one hundred fifty (150) were stocked in one pond (mean weight and length $1.48 \pm 0.46 \mathrm{~kg}$ and $57.31 \pm 6.42 \mathrm{~cm}$ ), one hundred fifty (150), distributed in three cages (mean weight and length $1.27 \pm 0.34 \mathrm{~kg}$ and $55.05 \pm 4.11 \mathrm{~cm}$ ). The fish were fed with extruded commercial ration with $15.0 \mathrm{~mm}$ floating pellets containing $40 \%$ crude protein (CP) and, 3,110Kcal of digestible energy $(D E)$, adjusted monthly. The parameters of water were: temperature $=24.08^{\circ} \mathrm{C} ; \mathrm{pH}=6.89 ;$ dissolved oxygen $=7.57 \mathrm{mg} \mathrm{L}^{-1}$. The values of mean final length $(P=74.07 \pm 4.34 \mathrm{~cm}$; $C=70.33 \pm 5.02 \mathrm{~cm})$ and final weight $(P=3.41 \pm 0.52 \mathrm{~kg}$; $C=2.94 \pm 0.60 \mathrm{~kg}$ ) they showed similar development in both systems. Means of factor condition - K - (0.009-0.036); daily weight gain $-D W G-\left(9.29 \mathrm{~g} \mathrm{day}^{-1}-8.95 \mathrm{~g}\right.$ day $\left.^{-1}\right)$; feed conversionFC - (3.09-4.15); Total ration consume - TRC - (29.6g day ${ }^{-1}$ $\left.74.16 \mathrm{~g} \mathrm{day}^{-1}\right)$; growing index - IC $-(0.219-0.215)$; survival $S-$ (97.33-90.67\%) for $P$ e C, respectively. Through the variance of analysis can be verified that there are significative interactions of the parameters between the culture systems and months $(P<0.05)$. The produced weight of fish was $R \$ 8.76$ (US\$ 2.85) and R\$ 8.73 (US\$2.33) for $P$ and C. Although $P$ showed best performance during the period and economic vantage, the growing index demonstrated that both systems had similar development.
\end{abstract}

Key words: Pseudoplatystoma corruscans, zootechnical and economic performances.

'Programa de Pós-Graduação em Aquicultura, Centro de Aqüicultura da UNESP (CAUNESP), Universidade Estadual Paulista (UNESP), Jaboticabal, SP, Brasil.

IIInstituto de pesca, Agência Paulista de Tecnologia dos Agronegócios (APTA), Água Funda, SP, Brasil. E-mail: eromagosa@pesca.sp.gov.br Autor para correspondência.

"I'Pólo APTA Leste Paulista, Água Funda, SP, Brasil. 


\section{INTRODUÇÃO}

O Pseudoplatystoma corruscans, conhecido regionalmente no Estado do Mato Grosso do Sul como pintado ou surubim (ordem dos Siluriformes, subordem Siluroidei, família Pimelodidae), pode ser encontrado nas principais bacias hidrográficas sul-americanas (PETRERE, 1995). Recentemente vem se adaptando à região Sudeste do país (SCORVO FILHO et al., 2004). Essa espécie apresenta carne de alta qualidade, de coloração clara e textura firme, com ausência de espinhos intramusculares, possibilitando ser oferecida ao consumidor em filés, inteiro ou eviscerado (TAVARES, 1997), sendo considerado um peixe nobre e apreciado no mercado nacional e internacional. Em função da excelente qualidade de sua carne e esportividade para pesca, o surubim vem sendo considerado um dos mais nobres e de maior valor comercial no Brasil (INOUE et al., 2009). Os autores afirmam que, no aspecto gastronômico, sua popularidade pode ser observada pelas inúmeras receitas regionais, as quais exaltam as características diferenciadas de sua carne (coloração clara, sabor suave e presença de poucos espinhos)

Os mercados das regiões Sul, Sudeste e Centro-Oeste consomem a maior parte de $\boldsymbol{P}$. corruscans comercializada no país. Praticamente todos são oriundos da pesca extrativista da região do Pantanal, Mato Grosso e Mato Grosso do Sul. Nos últimos anos, a oferta foi menor que a demanda, sendo necessária a importação de países vizinhos. Essas características apontam o grande potencial de expansão de mercado e abrem grandes perspectivas para os empreendimentos voltados a sua criação (KUBITZA et al., 1998).

O sistema semi-intensivo (viveiros escavados) tem sido mais utilizado por produtores da região Sul e Sudeste. Nesse modelo, com a adição fertilizantes químicos e adubos em grandes quantidades nos viveiros, promovendo a produtividade natural (ZIMMERMANN \& FITZSIMMONS, 2004). Um dos aspectos positivos desse sistema é a alta produção de algas, devido à oferta de maiores quantidades de ração, o que, por outro lado, provocando problemas na qualidade da água (SCORVO FILHO et al., 2004).

No sistema intensivo, destaca-se o uso de tanques-redes ou gaiolas como alternativa bastante difundida mundialmente, para produção de peixes em escala industrial (BJORNDAL, 1990; SCHMITTOU, 1997). Em tilápia do Nilo, Oreochromis niloticus (AYROZA et al., 2000), pacu, Piaractus mesopotamicus, e tambaqui, Colossoma macropomun (ONO, 2005) estudiosos verificaram que, no Brasil, ao longo da última década, a criação em tanques-rede se expandiu de forma expressiva, atribuindo a esse desenvolvimento os seguintes fatores: adaptações de pesquisas internacionais às nossas condições; utilização de altas densidades de estocagem por unidade de área ou volume; início da oferta de rações balanceadas. Os autores constataram a utilização de tanques-redes distintos quanto as formas e dimensões e, em diferentes fases de crescimento do peixe (recria e/ou engorda).

Embora a maioria das criações em tanquesrede seja feita em ambientes abertos, recentemente, MAINARDES-PINTO et al. (2003) recomendaram uma nova alternativa para a piscicultura intensiva, que possibilita o melhor aproveitamento da área inundada, como a utilização de tanques-rede de pequeno volume, instalados em viveiros povoados ou não com peixes.

O presente estudo teve como objetivo avaliar o desempenho produtivo de exemplares $\boldsymbol{P}$. corruscans estocados em dois sistemas: semi-intensivo (viveiros escavados) e intensivo (tanques-rede).

\section{MATERIAL E MÉTODOS}

Exemplares de Pseudoplatystoma corruscans (Siluriformes, Pimelodidae), resultantes de reprodução induzida, foram mantidos até um ano de idade, na Agência de Pesquisa e Tecnologia do Estado de São Paulo-APTA, no município de Pariquera-Açu, São Paulo, Brasil, (2443'S e 4753' 'W'), no período de janeiro de 2004 a janeiro de 2005.

Foram utilizados dois viveiros escavados na terra (VE), com aproximadamente $600 \mathrm{~m}^{2}$ cada de espelho d’água (12,0x50,0m), com profundida de média útil de $1,20 \mathrm{~m}$ e fluxo médio de água de $30 \mathrm{~L} \mathrm{~min}^{-1}$. Foram empregados dois sistemas de criação: viveiro escavado (VE) e tanque-rede (TR). Três tanques-rede foram instalados em um dos VE $(1,5 \times 1,5 \times 1,0 \mathrm{~m})$, totalizando $2,25 \mathrm{~m}^{3}$ cada, com abertura de malha de $2,0 \mathrm{~mm}$. Os TR foram cobertos com "sombrite" preto (40\%) para proporcionar um ambiente abrigado dos raios solares aos peixes cativos. Os peixes previamente anestesiados (benzocaína etil-aminobenzoato, diluída em $150 \mathrm{~mL}$ de álcool 96,0 $\mathrm{GL}$, em 20L de água, por 4 minutos) foram identificados (microchips) e, a seguir, pesados (kg) e medidos $(\mathrm{cm})$.

Os peixes foram divididos em dois lotes: (1) mantidos em VE (densidade de 1,0 peixe para cada $4 \mathrm{~m}^{2}$, totalizando 150 peixes) e (2) colocados em três TR (50 peixes por tanque-rede, totalizando 150 peixes). Esses peixes apresentaram, no início do experimento, os seguintes valores médios de peso e comprimento: $\mathrm{VE}=57,31 \pm 6,42 \mathrm{~cm} ; 1,48 \pm 0,46 \mathrm{~kg}$ e TR $=55,05 \pm 4,11 \mathrm{~cm} \mathrm{e}$ $1,27 \pm 0,34 \mathrm{~kg}$, respectivamente. 
Os peixes foram arraçoados, às $08 \mathrm{~h}$ e às $17 \mathrm{~h}$, com ração comercial extrusada, com teor de $40 \%$ PB.; $3110 \mathrm{kcal} \mathrm{ED} \mathrm{kg}^{-1} ; 7,0 \%$ de EE; $5,0 \%$ de matéria fibrosa; $20,0 \%$ de mineral e peletes de $15 \mathrm{~mm}$. A quantia de ração oferecida baseou-se na proporção da biomassa total, sendo de $1,5 \%$ (janeiro a maio/2004), $0,75 \%$ (junho a agosto/2004) e 1,8\% (setembro/2004 a janeiro/2005). Foram realizadas biometrias a cada 30 dias para acompanhar o ganho em crescimento e ajustar a quantidade de ração fornecida.

No final do experimento, 40 peixes (22VE e 6 de cada TR) foram sacrificados por sedação profunda (benzocaína). A seguir, foi realizada a incisão celomática para a retirada das vísceras e pesagem das gônadas e fígados. Macroscopicamente, foram identificados os sexos e classificados os estágios gonadais, segundo metodologia descrita por ROMAGOSA et al. (2003a).

Foram calculados os seguintes parâmetros: (1) relação peso $(\mathrm{Pt}) \mathrm{x}$ comprimento $(\mathrm{Ct})$ pela equação: $\mathrm{Pt}=\mathrm{axCt} \mathrm{b}$, em que: $\mathrm{a}=$ intersecção e $\mathrm{b}=$ coeficiente de alometria; (2) fator de condição (K) pelo coeficiente "b" da relação PtxCt, definido como o coeficiente de alometria; (3) ganho em peso diário (GPD=Pf-Pi)/t (Pf= peso médio final $(\mathrm{g}), \mathrm{Pi}=$ peso médio inicial $(\mathrm{g})$ e $\mathrm{t}=$ tempo em dias); (4) conversão alimentar (CA = CTR/GP (CTR = consumo total de ração e $\mathrm{GP}=$ ganho em peso); (5) índice de crescimento (G\% = In (PF) - In (PI) x 100/ tempo em dias, $\mathrm{PF}=$ peso final e $\mathrm{PI}=$ peso inicial; (6) sobrevivência (\%) pela relação porcentual entre o número de peixes no início e final do experimento.

Para a análise dos fatores físicos e químicos da água, foram coletadas amostras três vezes por semana ( $8 \mathrm{~h}$ e às $16 \mathrm{~h}$ ), sendo medidos (máxima e mínima) o oxigênio dissolvido (exceto fevereiro/04) e pH. Uma vez por semana, foram mensuradas a transparência da água e a concentração do teor de amônia.

Foi utilizado o programa SAS (1999) (Statistical Analysis System) para análise de variância dos parâmetros físicos e químicos da água e o teste de Tukey para comparação das médias. Para os outros parâmetros, foi utilizada a análise de variância, em parcelas subdivididas, verificando-se a diferença entre os sistemas de criação, meses e interação entre o sistema e mês e a comparação das médias pelos testes de Tukey e de Fisher, para verificar a proporção entre os sexos e tipo de tanque (ZAR, 1998).

No intuito de serem observados os gastos com ração em reais (R\$) e em dólares (US\$) (foi utilizado o valor dólar médio do ano de $2004=\mathrm{R} \$ 3,0751 \mathrm{US}^{-1}$ ), realizou-se a avaliação do consumo de ração e o valor pago pela mesma para cada viveiro. $\mathrm{O}$ preço de mercado para a ração utilizada, isto é, ração para carnívoro de $40 \%$ PB foi, em média, de R \$1,62 por quilo ao longo de todo o ensaio (US\$ $0,53 \mathrm{~kg}^{-1}$ ). Focaram-se apenas nos gastos com a ração, uma vez que foi o único parâmetro do custeio que variou entre os tratamentos. $\mathrm{Na}$ sequencia, calculou-se o Índice de Eficiência Econômica (IEE) e o Índice de Custo Médio, proposto por BARBOSA et al. (1992).

\section{RESULTADOS E DISCUSSÃO}

Nos dois sistemas, VE e TR, os valores médios de temperatura da água foram de $24,08 \pm 3,23^{\circ} \mathrm{C}$ e 24,08 $\pm 3,19^{\circ} \mathrm{C}$; oxigênio dissolvido $7,48 \pm 0,97 \mathrm{mg} \mathrm{L}^{-1} \mathrm{e}$

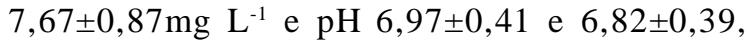
respectivamente. $\mathrm{OP}$. corruscans tolera bem a redução da temperatura da água durante o inverno, pois, mesmo quando se registraram médias mínimas de temperatura em torno de $11^{\circ} \mathrm{C}$, não houve registro de mortalidade (KUBITZA et al., 1998). O oxigênio dissolvido e pH se encontravam dentro de faixas satisfatórias para um desenvolvimento ótimo dos peixes (TAVARES, 1997; BURKERT, 2002). Os valores médios de transparência da água foram menores para o VE, diferindo dos primeiros meses, quando foram superiores aos dos TR. Os índices de amônia estiveram abaixo de 1,0ppm durante o experimento.

$\mathrm{Na}$ figura 1, pode-se observar o desempenho em peso e comprimento durante o período experimental. Verifica-se que as curvas de crescimento em peso e comprimento foram significativamente superiores nos peixes do VE. O crescimento dos peixes mantidos em TR foi inferior provavelmente devido à restrição de espaço, embora os animais se mantivessem sadios e sem lesões, indicando boa sanidade. Por outro lado, uma alta densidade de estocagem pode causar grande mortalidade e redução do crescimento, uma vez que a decomposição do excesso de alimento e os resíduos nitrogenados provenientes da excreção dos peixes podem prejudicar a qualidade da água (JOBLING, 1994).

A curva ajustada de peso e comprimento do VE e TR, $\mathrm{Pt}=9 \times 10^{-7} \mathrm{Ct} 3,50$ e $\mathrm{Pt}=4 \times 10^{-7} \mathrm{Ct} 3,75$, respectivamente, evidenciou crescimento alométrico. Em P. fasciatum, mantidos nos VE na mesma região, obtiveram o valor de "b" inferior (machos 3,128 e fêmeas 3,484) (ROMAGOSA et al., 2003b). Em geral, as informações encontradas na literatura referem-se à fase de alevinos até atingirem aproximadamente $1,0 \mathrm{~kg}$, quando recebem rações comerciais com peletes de diâmetro de até $8 \mathrm{~mm}$ (BURKET, 2002). Entretanto, KUBITZA et al. (1998) utilizaram ração flutuante com diâmetro variando entre 10 a $15 \mathrm{~mm}$ na alimentação de Pseudoplatystoma ssp no período de finalização $(\geqslant 3,0 \mathrm{~kg})$, estocados em tanques de terra de 6,0ha, em uma densidade em torno de 1.650 peixes ha ${ }^{-1}$. 


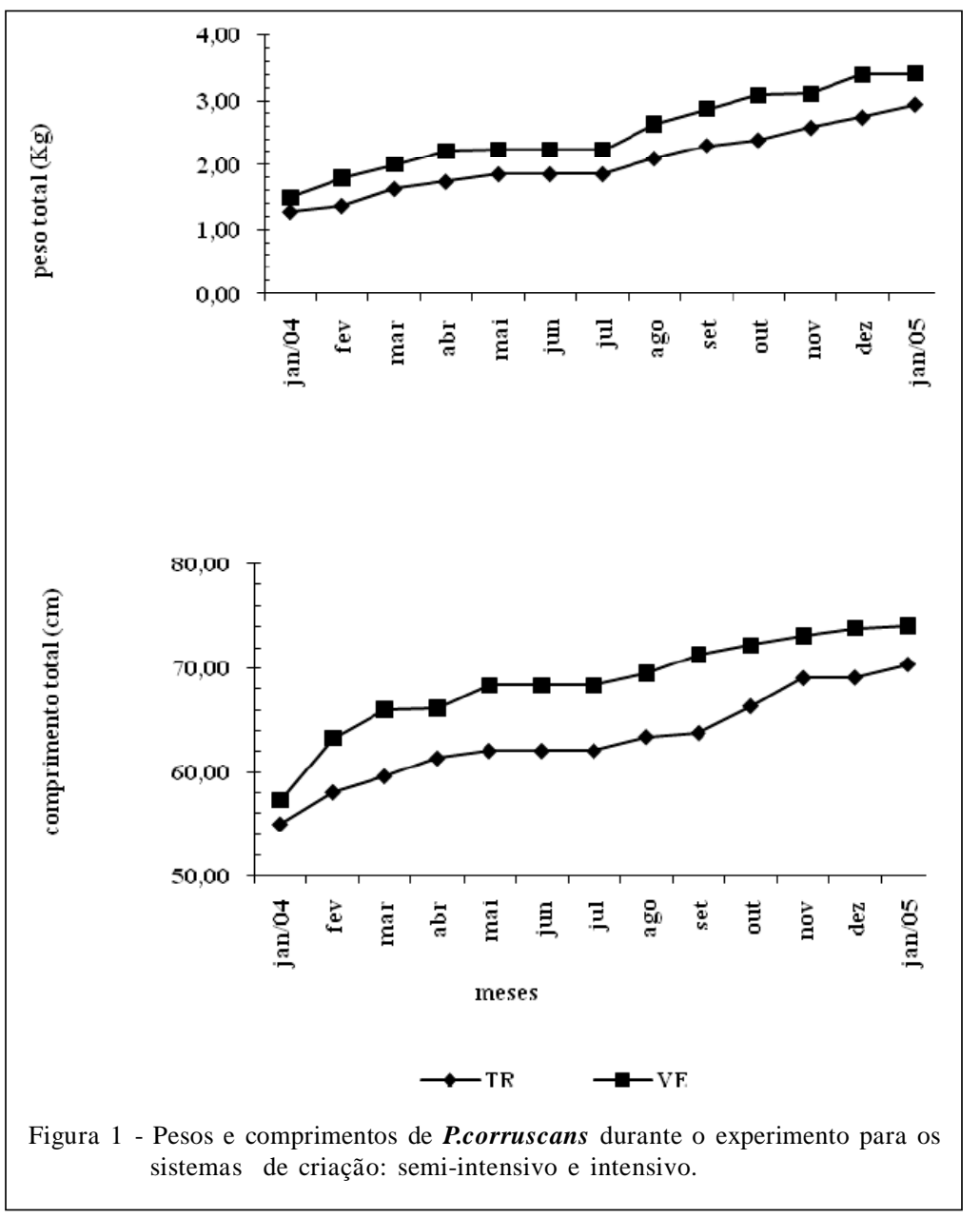

Em ambiente natural, MIRANDA \& RIBEIRO (1997) e GODINHO et al. (1997), estudando exemplares da mesma espécie, capturados no Rio São Francisco, MG, encontraram valores de "b" de 3,52 e 3,33, respectivamente. Esses resultados são ligeiramente inferiores, comparando-os aos de MATEUS \& PETRERE JR (2004), que verificaram valor de "b" inferior a 3,13 em $\boldsymbol{P}$. corruscans capturados na bacia do rio Cuiabá, Pantanal de Mato Grosso. Neste estudo, os peixes mantidos em TR apresentaram valores de "b" ligeiramente superiores $(b>3,8)$.

Os valores de K para $\boldsymbol{P}$. corruscans foram ligeiramente superiores no $\mathrm{VE}(0,08-0,10)$, em relação aos TR $(0,03-0,04)$. Pela análise de variância, verificouse interação significativa entre os sistemas de criação e o mês (Tabela 1). Os valores de $\mathrm{K}$ apresentaram pequenas variações para os peixes mantidos em VE, provavelmente, isso ocorreu devido à maior dispersão da ração, uma vez que os animais encontravam-se soltos, facilitando a procura por alimento.
A ração oferecida (diâmetro pelete $=15 \mathrm{~mm}$; $3110 \mathrm{kcal}^{\mathrm{de} E D \mathrm{~kg}^{-1} \mathrm{e}} 40 \% \mathrm{~PB}$ ) apresentava a quantidade $\mathrm{ED} / \mathrm{PB}$ recomendada para a fase de crescimento e indicada por CYRINO (2000). Contudo, BURTLE (1990), estudando bagre do canal, utilizou índices menores de ED/PB (2700-2800ED kg-1 e 24-26\%PB), obtendo resultados promissores.

Para $\boldsymbol{P}$. corruscans, os valores médios de GPD e CAA (Tabelas 1 e 2) foram melhores para o VE em comparação aos do TR. Esses resultados corroboram os de KUBITZA \& CYRINO (1997). Os autores sugerem que para se obter menores índices é preciso ter conhecimento prévio de fatores, como: espécie, idade, manejo alimentar, qualidade da água e densidade de estocagem.

Houve interação significativa entre as variáveis GP e CAA, os sistemas de criação e mês. Experimentos com $\boldsymbol{P}$. corruscans de mesma idade foram descritos por MELO et al. (2003), que estimaram médias de GPD inferiores (2,3g dia $\left.{ }^{-1}\right)$, quando comparados aos 
Tabela 1 - Média e desvio padrão dos parâmetros zootécnicos analisados durante o experimento para $\boldsymbol{P}$. corruscans em função dos sistemas de criação: semi-intensivo e intensivo.

\begin{tabular}{lcc}
\hline Parâmetros & VE & TR \\
\hline Peso inicial (kg) & $1,448^{\mathrm{a}}$ & $1,269^{\mathrm{a}}$ \\
Peso final (kg) & $3,411^{\mathrm{a}}$ & $2,942^{\mathrm{b}}$ \\
Peso x Comprimento & $3,5008^{\mathrm{a}}$ & $3,7509^{\mathrm{a}}$ \\
Fator de Condição (K) & $0,09^{\mathrm{a}}$ & $0,03^{\mathrm{b}}$ \\
Ganho de Peso Diário (GPD g dia & \\
Conversão Alimentar Aparente (CAA) & $9,291^{\mathrm{a}}$ & $8,950^{\mathrm{a}}$ \\
Consumo de Ração $\left(\mathrm{g} \mathrm{dia}{ }^{-1}\right)$ & $3,09^{\mathrm{a}}$ & $4,15^{\mathrm{a}}$ \\
Sobrevivência Final (S, \%) & $29,60^{\mathrm{b}}$ & $24,72^{\mathrm{a}}$ \\
\hline
\end{tabular}

Médias seguidas na mesma letra não diferem entre si pelo teste de Tukey $(\mathrm{P}>0,05)$. Peso $(\mathrm{CV}=22,41 \%)$; $\mathrm{K}(\mathrm{CV}=4,93 \%)$; $\mathrm{GPD}(\mathrm{CV}=17,7 \%)$; CAA (CV=19,8\%); Consumo de ração $(\mathrm{CV}=8,53 \%)$ e Sobrevivência $(\mathrm{CV}=1,32 \%)$.

valores médios de GPD (TR=8,95g dia ${ }^{-1}$ VE=9,29g dia $\left.{ }^{-1}\right)$ deste estudo.

O consumo de ração total foi superior para os peixes mantidos em $\mathrm{VE}$, quando comparados aos do TR (Tabela 2). Entretanto, a dificuldade de acesso ao alimento para os peixes mantidos em VE provavelmente seja maior; ainda que a ração tenha sido ofertada adequadamente e em quantidades compatíveis com sua biomassa, deve ter ocorrido competição entre os animais e/ou a perda da ração. Fato este comprovado pelos valores médios de consumo total de ração (Tabelas 1 e 2).

As taxas médias finais de sobrevivência foram de 97,33 e 90,67\% nos VE e TR, respectivamente, com diferenças significativas de 5\% entre os sistemas de criação. No caso dos TR, os resultados foram superiores aos descritos por BURKERT (2002), observado para $\boldsymbol{P}$. corruscans em torno de $67,9 \%$, atribuindo-se as perdas ao estresse térmico e ao canibalismo. As taxas de mortalidade provavelmente foram causadas pelo manejo dos animais durante as biometrias (setembro TR e outubro VE).

Fazendo uma análise econômica, o insumo 'ração' é visto como o de maior custo de produção (SCORVO FILHO, 1998, 1999). Nesse caso, pelas características do experimento, o insumo ração foi o item que apresentou variação no custo operacional de produção. Os outros itens (alevinos, corretivos e desinfetantes de solo), bem como mão-de-obra, não variaram entre os tratamentos, istoé, no VE com peixes soltos e nos três tanques-rede presos (Tabela 3).

Tabela 2 - Médias mensais do Fator de Condição (K), Ganho de Peso Diário (GPD) e Conversão Alimentar Aparente (CAA) durante o período experimental dos sistemas de criação: semi-intensivo e intensivo.

\begin{tabular}{|c|c|c|c|c|c|c|}
\hline \multirow{3}{*}{ Período } & \multicolumn{6}{|c|}{ 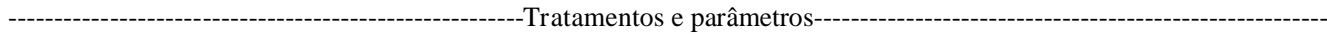 } \\
\hline & \multicolumn{2}{|c|}{ 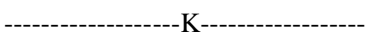 } & \multicolumn{2}{|c|}{-------------GPD g dia ${ }^{-1}$------------ } & \multicolumn{2}{|c|}{ 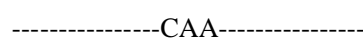 } \\
\hline & VE & TR & VE & TR & VE & TR \\
\hline 1 & $0,100^{\text {A a }}$ & $0,038^{\mathrm{B} \mathrm{bc}}$ & $13,48^{\text {A a }}$ & $5,47^{\mathrm{Bcd}}$ & $1,48^{\mathrm{Aa}}$ & $2,47^{\mathrm{A} a b}$ \\
\hline 2 & $0,085^{\mathrm{Ac}}$ & $0,033^{\mathrm{Bd}}$ & $8,63^{\mathrm{Abc}}$ & $11,14^{\mathrm{Ab}}$ & $2,63^{\mathrm{Aab}}$ & $2,08^{\mathrm{A} a b}$ \\
\hline 3 & $0,082^{\mathrm{Ac}}$ & $0,035^{\text {B cd }}$ & $8,16^{\mathrm{Abc}}$ & $3,51^{\mathrm{Bd}}$ & $3,59^{\mathrm{A} \text { ab }}$ & $7,31^{\text {B d }}$ \\
\hline 4 & $0,091^{\mathrm{Ab}}$ & $0,034^{\mathrm{Bd}}$ & $4,86^{\mathrm{Ac}}$ & $4,49^{\mathrm{Ad}}$ & $3,57^{\mathrm{A} \text { ab }}$ & $3,02^{\mathrm{Abc}}$ \\
\hline 5 & $0,082^{\mathrm{Ac}}$ & $0,035^{\mathrm{Bcd}}$ & - & - & - & - \\
\hline 8 & $0,091^{\mathrm{Ab}}$ & $0,044^{\text {В а }}$ & $10,83^{\mathrm{A} a b}$ & $8,94^{\mathrm{Abc}}$ & $1,23^{\mathrm{Aa}}$ & $1,52^{\mathrm{Aa}}$ \\
\hline 9 & $0,090^{\mathrm{Ab}}$ & $0,039^{\mathrm{B} \mathrm{b}}$ & $9,40^{\mathrm{A} a b c}$ & $3,27^{\mathrm{Bd}}$ & $4,26^{\mathrm{Abc}}$ & $9,68^{\mathrm{B} \mathrm{e}}$ \\
\hline 10 & $0,093^{\mathrm{Ab}}$ & $0,037^{\mathrm{B} \mathrm{bc}}$ & $7,11^{\mathrm{B} \mathrm{bc}}$ & $11,60^{\mathrm{Ab}}$ & $7,55^{\mathrm{Bd}}$ & $4,01^{\mathrm{Ac}}$ \\
\hline 11 & $0,093^{\mathrm{Ab}}$ & $0,039^{\mathrm{B} \mathrm{b}}$ & $9,47^{\mathrm{A} a \mathrm{abc}}$ & $7,22^{\mathrm{Ac}}$ & $5,67^{\mathrm{A} \mathrm{dcd}}$ & $6,07^{\mathrm{Ad}}$ \\
\hline 12 & $0,094^{\mathrm{A} \mathrm{b}}$ & $0,035^{\mathrm{B} \mathrm{cd}}$ & $7,57^{\mathrm{A} \mathrm{bc}}$ & $11,15^{\mathrm{Ab}}$ & $7,09^{\mathrm{Ad}}$ & $4,04^{\mathrm{Ac}}$ \\
\hline 13 & $0,094^{\mathrm{A} \mathrm{b}}$ & $0,035^{\mathrm{B} \mathrm{cd}}$ & $13,37^{\text {В а }}$ & 22,71 A а & $2,76^{\mathrm{Aab}}$ & $1,35^{\mathrm{A} \mathrm{a}}$ \\
\hline
\end{tabular}

Médias seguidas da mesma letra minúscula na coluna e maiúscula na linha não diferem entre si pelo teste de Tukey (P>0,05).

Ciência Rural, v.41, n.3, mar, 2011. 
Tabela 3 - Consumo de ração em quilo; gastos com ração em real e dólar; produção de peixes em quilo de ração, produção de peixes em quilo e custo do quilo de peixe em real e dólar ${ }^{*}$, durante o período experimental dos sistemas de criação: semi-intensivo e intensivo.

\begin{tabular}{lcccccc}
\hline Sistema & $\begin{array}{c}\text { Consumo ração } \\
(\mathrm{kg})\end{array}$ & $\begin{array}{c}\text { Gastos ração } \\
(\mathrm{R} \$)\end{array}$ & $\begin{array}{c}\text { Gastos ração } \\
(\mathrm{US} \$)\end{array}$ & $\begin{array}{c}\text { Produção } \\
(\mathrm{kg})\end{array}$ & $\begin{array}{c}\text { Custo kg peixe } \\
\left(\mathrm{R} \$ \mathrm{~kg}^{-1}\right)\end{array}$ & $\begin{array}{c}\text { Custo Kg peixe } \\
\left(\mathrm{US} \$ \mathrm{~kg}^{-1}\right)\end{array}$ \\
\hline VE & $1.831,6$ & $2.967,19$ & 964,91 & 560,69 & 5,29 & 1,72 \\
TR & $1.494,67$ & $2.421,37$ & 787,41 & 467,76 & 5,18 & 1,68 \\
\hline
\end{tabular}

*Dólar médio do ano de 2004 - US\$1 = R \$3,0751.

O Índice de Eficiência Econômica e o Índice de Custos Médios demonstraram os melhores resultados para a criação de pintados em TR, nos quais o IEE foi 100 para TRe 101,9 para VE. O Índice de Custo Médio foi de 98,1 para os peixes em VE e 100 para aqueles em TR. Tais valores mostram que o melhor sistema de criação, em termos de índice de eficiência econômica e de custo de ração, foi a criação em TR. O custo médio por quilo de peixe vivo também foi melhor para aqueles criados em TR. Tais valores representaram uma vantagem econômica de 1,9\%, de acordo com sistema empregado.

\section{CONCLUSÃO}

Exemplares de $\boldsymbol{P}$. corruscans que receberam ração comercial (extrusada), flutuante de $15 \mathrm{~mm}$, teores de $40 \%$ PB e $3.110 \mathrm{kcal} \mathrm{ED} \mathrm{kg}^{-1}$ de alimento e estocados nas condições climáticas da região do Vale do Ribeira, apresentaram, no sistema semi-intensivo (VE), parâmetros estimados elevados e satisfatórios ( $\mathrm{Pt}$ x Ct; GPD e CAA), em comparação aos do sistema intensivo (TR). Entretanto, uma vez que no início do experimento os peixes do VE tiveram peso inicial superior quando comparados aos do TR, o índice de crescimento revela valores de desempenho igual para os dois sistemas, não mostrando benefício no VE. Todavia, a criação em TR apresentou vantagem econômica de 1,9\%.

\section{AGRADECIMENTOS}

À Coordenação de Aperfeiçoametnto de Pessoal de Nível Super (CAPES), pela bolsa concedida durante a realização do Mestrado no Centro de Aquicultura da Universidade Estadual Paulista (CAUNESP), Jaboticabal, São Paulo, SP. Aos técnicos de apoio à pesquisa, Benedito Martins de Aguiar, Célio Martins de Aguiar, Edilberto Rufino de Almeida, do Pólo Regional do Vale do Ribeira, pelo apoio no manejo dos viveiros. À colega, Dr Vanessa Xavier Linhares de Andrade, CAUNESP, Jaboticabal, $\mathrm{SP}$, que colaborou intensamente durante o experimento.

\section{REFERÊNCIAS}

BARBOSA, H.P. et al. Triguilho para suínos nas fases iniciais de crescimento, crescimento e terminação. Revista Brasileira de Zootecnia, Minas Gerais, v.21, p.827-837, 1992.
AYROZA, L.M. et al. Desempenho da tilápia do Nilo, Oreochromis niloticus, em tanques-rede, em empresa rural. In: SIMPÓSIO BRASILEIRO DE AQUICULTURA, 11., 2000, Florianópolis, SC. Anais... Florianópolis: SIMBRAq, 2000. Disponível em CD.

BJORNDAL, T. The economics of salmon aquaculture. London and Boston: Blackwell Scientific Publications Oxford (United Kingdom), 1990. 119p.

BURKERT, D. Cultivo do surubim em tanques-redes com três rações para peixes carnívoros. 2002. 78f. Dissertação (Mestrado em Produção Animal) - Centro de Ciência e Tecnologia Agropecuária, Universidade do Norte Fluminense, Goytacazes, Rio de Janeiro, RJ.

BURTLE, G.J. Body composition of farm-raised catfish can be controlled by attention to nutrition. Feedstuffs, v.62, p.6871,1990 .

CYRINO, J.E.P. Condicionamento alimentar e exigência nutricional de espécies carnívoras. 2000. 200f. Livre Docência. Texto sistematizado apresentado na Especialidade Aqüicultura. ESALQ/USP, Piracicaba, São Paulo, SP.

GODINHO, H.P. et al. Pesca e biologia do surubim Pseudoplatystoma corruscans no Rio São Francisco. In: MIRANDA, M.O.T. (Org.). Surubim. Belo Horizonte: IBAMA, 1997. p.27-42 (Coleção Meio Ambiente, Série Estudos Pesca, 19).

JOBLING, M. Fish bionergetics. London: Chapman \& Hall, 1994. 309p.

INOUE, L.A.K. et al. Princípios básicos para produção de alevinos de surubins (Pintado e Cachara). Dourados: Embrapa Agropecuária Oeste; Manaus: Embrapa Amazônia Ocidental; Corumbá: Embrapa Pantanal, 2009. 26p. (Documentos/ Embrapa Agropecuária Oeste, ISSN 1516-845X; 99; Documentos / Embrapa Amazônia Ocidental; ISSN 1517-3135; 68; Documentos / Embrapa Pantanal, ISSN 1517-1973;100).

KUBITZA, F., CYRINO, J.E.P. Feed training strategies for the piscivoros peacock bass Cichla sp. In: INTERNATIONAL SYMPOSIUM BIOLOGY OF TROPICAL FISHES, 1997, Manaus, AM. Proceedings... Manaus: Instituto Nacional de Pesquisas da Amazônia, Manaus, 1997. p.139.

KUBITZA, F. et al. Produção Intensiva no Projeto Pacu Ltda.e Agropeixe Ltda. Panorama da Aqüicultura, v.8, p.41-49, 1998. 
MAINARDES-PINTO, C.S.R. et al. Viability of Thailand tilapia, Oreochromis niloticus culture raised in small volume net cages placed in populated ponds. In: WORLD AQUACULTURE 2003, Salvador, Bahia, BA. Book of Abstracts... Salvador: WAS, 2003. p.442.

MATEUS, L.A.F., PETRERE, Jr.M. Age, growth and yield per recruit analysis of the pintado Pseudoplatystoma corruscans (Agassiz, 1829) in the Cuiabá River Basin, Pantanal Matogrossense, Brazil. Brazilian Journal Biology, v.64, n.2, p. 257-264, 2004.

MELO, D.C. et al. Growth of surubim Pseudoplatystoma corruscans at different stock density. In: WORLD AQUACULTURE SOCIETY, 2003, Salvador, Bahia, BA. Book of Abstracts... Salvador: WAS, 2003. p.481.

MIRANDA, M.O.T. RIBEIRO, P. L. Características zootécnicas do surubim. In: MIRANDA, M.O.T. (Org). Surubim. Belo Horizonte: IBAMA, 1997. p.43-56. (Coleção Meio Ambiente. Série Estudos Pesca).

ONO, E.A. Criação de peixes em tanques-rede. In: ZOOTEC, 2005, Campo Grande, MS. Anais... Campo Grande: Zootec, 2005. p.1-14.

PETRERE Jr, M. A pesca de água doce no Brasil. Ciência Hoje, v.19, p.28-33, 1995.

ROMAGOSA, E. et al. Biologia reprodutiva de fêmeas de cachara, Pseudoplatystoma fasciatum (Teleostei, Siluriformes, Pimelodidae), mantidas em cativeiro. Boletim do Instituto de Pesca, São Paulo, v.29, n.2, p.151-159, 2003a.

ROMAGOSA, E. et al. Características morfométricas e crescimento do cachara, Pseudoplatystoma fasciatum em cativeiro. Acta Scientarium, v.2, p.277-283, 2003b.
SAS Institute Inc. User's guide. Statistic. SAS/GRAPH Software: Reference. Cary, NC: SAS Institute Inc. 1999. Version 8.

SCHMITTOU, H.R. Produção de peixes em alta densidade em tanques-redes de pequeno. Campinas: Mogiana Alimentos e Associação Americana de Soja, 1997. 78p.

SCORVO FILHO, J.D. Aspectos econômicos da piscicultura de água doce com ênfase na cadeia produtiva. In: SIMPÓSIO SOBRE MANEJO E NUTRIÇÃO DE PEIXES, 2., 1998, Piracicaba, SP. Anais... Piracicaba: Colégio Brasileiro de Nutrição Animal, 1998. p.21-34.

SCORVO FILHO, J.D. Avaliação técnica e econômica das piscigranjas de três regiões do estado de São Paulo. 1999. 98f. Tese (Doutorado em Aqüicultura) - Centro de Aqüicultura da UNESP, Universidade Estadual Paulista, Jaboticabal. SP. Disponível em: <http://www.caunesp.unesp.br>. Acesso em: 02/01/2006.

SCORVO FILHO, J.D. et al. Desempenho do pintado, Pseudoplatystoma corruscans (Spix \& Agassiz, 1829) criado nos sistemas intensivo e semi-intensivo. In: CONGRESSO DA AQUABIO AQUIMERCO, 2004, Vitória, ES. Anais... Vitória: Aqua Ciência, 2004. 145p.

TAVARES, M.P. O surubim. In: MIRANDA, M.O.T. (Org.). Surubim. Belo Horizonte: IBAMA, 1997. p. 9-25. (Coleção Meio Ambiente. Série Estudos Pesca, 19).

ZAR, J. Biostatistical analysis. 4.ed. New Jersey: PrenticeHall, 1998. 930p.

ZIMMERMAM, S. FITZSIMMONS, K. Tilapicultura intensiva. In: CYRINO, J.E.P. et al. (Ed.). Tópicos especiais em piscicultura de água doce tropical intensiva. São Paulo: Sociedade Brasileira de Aqüicultura e Biologia Aquática. TecArt, 2004. Cap.9, p.239-266. 